This information is current as of April 26, 2023.

\title{
Cerebral Vasospasm: New Strategies in Research and Treatment
}

AJNR Am J Neuroradiol 2009, 30 (7) e105-e106 doi: https://doi.org/10.3174/ajnr.A1611

http://www.ajnr.org/content/30/7/e105 


\section{BOOK REVIEW}

\section{Cerebral Vasospasm: New Strategies in Research and Treatment}

T. Kiriş and J.H. Zhang, eds. Springer Wien; 2008, 450 pages, $\$ 289.00$.

C erebral vasospasm after aneurysmal subarachnoid hemorrhage (SAH) is one of the most difficult problems encountered by the clinicians who take care of these critically ill patients. The etiology of cerebral vasospasm after SAH has been firmly established as a subarachnoid blood clot. Cerebral Vasospasm: New Strategies in Research and Treatment serves well the functions of briefly recapitulating the established knowledge about cerebral vasospasm as it relates to $\mathrm{SAH}$, while also delving deeper into new and ongoing areas of active research in the understanding of delayed ischemic neurologic deficits and cerebral vasospasm.

The editors have organized the book as a collection of individual journal articles assembled by topic into 12 sections. This arrangement helps to sort a tremendously complex array of articles into a more manageable series of discussions. These 12 sections examine cerebral vasospasm pathogenesis, biochemistry, electrophysiology, pharmacology, molecular biology, vasospasm vascular remodeling, vasospasm diagnosis, medical treatment, chemical surgery, surgical treatment, prognosis, and other areas of vasospasm research. Each section assembles a number of articles that cover various aspects of the knowledge in that particular area.

The first section discusses several current avenues of active research in the pathogenesis of cerebral vasospasm after SAH. These avenues include apoptotic signal-intensity-transduction pathways, oxidative stress from free radicals and other oxidative products, as well as myofibroblastic changes in cerebrovascular smooth muscle cells. These few articles cover the broad area well, touching on many advances in pathogenesis as well as current understanding and ongoing research. This section also serves as a useful introduction to the many subsequent articles in closely related areas of scientific inquiry.

The section on pathogenesis transitions smoothly into the following sections that cover biochemistry, electrophysiology, pharmacology, molecular biology, and vasospasm vascular remodeling. A very appropriate emphasis is placed on mitogenic, inflammatory, and neuroprotective signal-intensitytransduction pathways. There is a very adequate review of research pertaining to calcium-channel blockers, statins, endothelin, and nitric oxide, because these topics are the mainstay of current treatments as well as areas that may prove to be the best new prospects for drug-development arenas. The section on vasospasm remodeling introduces this issue as another pathophysiologic disturbance within the spectrum of the disease and, therefore, offers an opportunity for further research. A strong emphasis on basic science research advances in these first several sections makes them suitable for clinicians and basic researchers alike.

At roughly the midpoint of the text, the sections shift from primarily a basic science slant to a more clinical one. The first of these sections involves vasospasm diagnostics and begins with novel technologies and innovative uses of more traditional ones and ends with the widely used angiography, MR imaging perfusion/diffusion imaging, and transcranial Doppler sonography. This section only lightly covers the current standards of care and introduces a select few new ideas. For those keenly interested in the diagnostic techniques and innovations in va-

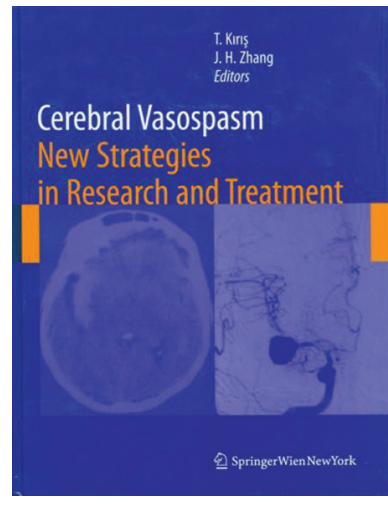
sospasm research, this section references areas for further reading.

Vasospasm medical treatment is well covered, particularly when combined with the prior sections that discuss electrophysiology and pharmacology. Inclusion in this section of a discussion of magnesium, statins, and other agents shows the limited repertoire currently available and the true need for improved knowledge and treatment modalities.

The section on chemical surgery taken as a whole makes a persuasive argument for more aggressive intraoperative and postoperative clearance of subarachnoid clot to prevent, not just treat, cerebral vasospasm. The use of urokinase and cisternal irrigation is strongly advocated in these articles. The addition of urokinase as an adjunct to crystalloid irrigation renews an old discussion in a new light.

Surgical treatments are briefly discussed in an article on decompressive craniectomy, followed by a series of articles on endovascular treatment of vasospasm, a great advance during the past several years. These articles review the evidence demonstrating the need for aggressive angioplasty and intra-arterial calcium-channel blocker use to treat moderate-to-severe vasospasm resistant to "triple-H" therapy. A good understanding of the critical role endovascular therapy plays in these situations cannot be emphasized enough, but this role is adequately covered in this section.

The penultimate series of articles covers the prognoses of patients who have SAH and are likely to develop cerebral vasospasm. In just a few articles, the spectrum of factors involved is thoroughly covered, and some fresh ideas for markers of vasospasm are also introduced. The combination of review of old and introduction of new will interest a large number of readers.

The final section of the text includes a series of articles discussing vasospasm in other settings, such as in pediatrics and posttrauma and after illicit substance use. These last several articles serve to remind the reader that clinicians do, rarely, encounter vasospasm in atypical situations in which suspicion and timely treatment make a difference in the outcome.

Overall, the collection of articles in this book covers the broad and complex field of cerebral vasospasm after SAH. It is comprehensive in breadth and depth, including coverage of both basic and clinical science and review of old and introduction of new topics, and achieves this with generally well- 
written content. The areas that seem to be beyond the scope of a single book are well-referenced and discussed appropriately, nonetheless. As a whole, this book is an accurate and current source for any physician who is likely to encounter patients with SAH, including neurologists, neurosurgeons, neurointensivists, neuroradiologists, interventionalists, and researchers in these fields.

DOI 10.3174/ajnr.A1611 